\title{
Serine-Arginine Protein Kinase-like Protein, SrpkF, Stimulates Both Cellobiose-responsive and D- Xylose-Responsive Signaling Pathways in Aspergillus Aculeatus
}

\section{Ryohei Katayama}

Osaka Prefecture University

Natsumi Kobayashi

Osaka Prefecture University

Takashi Kawaguchi

Osaka Prefecture University

Shuji Tani ( $\sim$ shuji@biochem.osakafu-u.ac.jp )

Osaka Prefecture University https://orcid.org/0000-0003-3444-9482

\section{Research Article}

Keywords: Gene regulation, Cellulase, ManR, XInR, filamentous fungi, phosphorylation

Posted Date: July 13th, 2021

DOl: https://doi.org/10.21203/rs.3.rs-691475/v1

License: (1) (1) This work is licensed under a Creative Commons Attribution 4.0 International License. Read Full License

Version of Record: A version of this preprint was published at Current Genetics on August 28th, 2021. See the published version at https://doi.org/10.1007/s00294-021-01207-x. 


\title{
Serine-arginine protein kinase-like protein, SrpkF, stimulates both cellobiose-responsive and D- xylose-responsive signaling pathways in Aspergillus aculeatus
}

Ryohei Katayama, Natsumi Kobayashi, Takashi Kawaguchi, Shuji Tani*

Graduate School of Life and Environmental Sciences, Osaka Prefecture University, 1-1 Gakuen-cho, Sakai, Osaka 599-8531, Japan

\section{* Corresponding author}

The corresponding author is Shuji Tani. e-mail: shuji@biochem.osakafu-u.ac.jp

ORCID: 0000-0003-3444-9482

\begin{abstract}
To elucidate the regulatory mechanisms of various cellulolytic enzyme genes in Aspergillus aculeatus, we identified one mutant that reduced the expression of FIII-avicelase $(c h b I)$ in response to cellulose from 12,000 A. aculeatus T-DNA-inserted mutants. The T-DNA inserted into a putative protein kinase gene similar to AN10082 in A. nidulans, the serine-arginine protein kinase F, SrpkF. The fold increase in

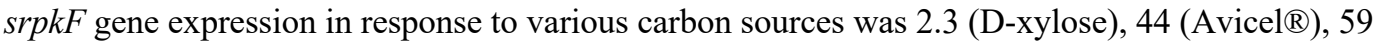
(Bacto $^{\mathrm{TM}}$ Tryptone), and 98 (no carbon) compared with D-glucose. The deletion of srpkF in A. aculeatus resulted in a significant reduction in the cellulose-responsive expression of $c h b I$, hydrocellulase $(c e l 7 b)$, and FIb-xylanase $(x y n I b)$ genes at an early induction phase. However, the srpkF deletion did not affect the expression of $x y n I b$ in response to D-xylose. Furthermore, the $\operatorname{srpkF}$-overexpressing strain that expresses the $\operatorname{srpkF}$ gene at levels from four- to nine-fold higher than the control strain stimulated the expression of $c b h I$ and $c e l 7 b$ in response to cellobiose and the FI-carboxymethyl cellulase gene $(\mathrm{cmcl})$ and $x y n I b$ in response to xylose. The expression of $c b h I$ and $c e l 7 b$ is regulated by a transcriptional activator, ManR, and the expression of $c m c 1$ and $x y n I b$ is regulated by XlnR. Our data demonstrate that SrpkF can stimulate both the ManR-and XlnR-dependent signaling pathways in response to cellobiose and D-xylose in A. aculeatus.
\end{abstract}

\section{Keywords:}

Gene regulation, Cellulase, ManR, XlnR, filamentous fungi, phosphorylation

\section{Declarations}

Funding 
This work was supported by JSPS KAKENHI under Grant 19K05777. Part of this work was also supported by the New Energy and Industrial Technology Development Organization (NEDO) Project under Grant P07015.

\section{Conflicts of interest}

The authors have no conflicts of interest to declare that are relevant to the content of this article.

\section{Availability of data and material}

All data generated or analyzed during this study are included in this published article and its Supplementary Information files. DNA sequences will be available at the DDBJ database when this article is released for publication.

\section{Code availability}

Not applicable.

\section{Author contributions}

S.T. and T.K. conceived and designed the project. R.K. and N.K. conducted the experiments. S.T wrote the paper. All authors read and approved the final manuscript.

\section{Ethics approval}

This article does not contain any studies that involve human participants or animals.

\section{Consent to participate}

Not applicable.

\section{Consent for publication}

Not applicable.

\section{Acknowledgments}

This work was supported by Grants-in-Aid for Scientific Research from Japan Society for the Promotion of Science under Grant 19K05777. Part of this work was also supported by the New Energy and Industrial Technology Development Organization Project under Grant P07015. 


\section{Introduction}

Filamentous fungi are prominent producers of enzymes that degrade lignocellulose (Payne et al. 2015). Among filamentous fungi, Trichoderma reesei is well known to produce copious amounts of cellulolytic enzymes (Bischof et al. 2016). However, T. reesei glycoside hydrolases are being continuously improved to utilize lignocellulose for feedstock and generate biobased products. For example, $\beta$-glucosidase from Aspergillus aculeatus no. F-50 [NBRC 108789] was introduced into T. reesei to supplement its activities, which accelerated cellulose hydrolysis (Baba et al. 2015; Nakazawa et al. 2012). $\beta$-Glucosidase from $A$. aculeatus showed high compatibility in T. reesei, suggesting that A. aculeatus produces promising enzymes to liberate glucose from cellulose. However, cellulolytic enzymes of A. aculeatus are not utilized in industry because of low production levels. We aim to understand the regulatory mechanisms of the associated gene expressions and apply this knowledge to improving enzyme production in $A$. aculeatus.

Cellulolytic enzyme production in Aspergillus is regulated at the transcriptional level. The first identified transcriptional activator of cellulolytic and xylanolytic genes was XlnR, a Zn(II) ${ }_{2}$ Cys6-type transcriptional activator that coordinates xylanolytic expression in Aspergillus niger (van Peij et al. 1998). Genetic analysis revealed that XlnR controls the expression of not only xylanolytic enzyme genes but also cellulolytic enzyme genes in A. aculeatus (Kunitake and Kobayashi 2017; Tani et al. 2014). XlnR regulates the expression of FIb-xylanase $(x y n I b)$ and FI-carboxymethyl cellulase $(\mathrm{cmcl})$ genes, whereas FIII-avicelase $(c b h I)$ and FII-carboxymethyl cellulase $(c m c 2)$ are under the control of the XlnRindependent signaling pathway in A. aculeatus (Tani et al. 2012). Clr-2 in Neurospora crassa and ClrB in Aspergillus nidulans were identified as $\mathrm{Zn}(\mathrm{II})_{2} \mathrm{Cys} 6$-type transcriptional activators controlling the expression of cellulolytic enzyme genes in response to cellulosic carbon sources (Coradetti et al. 2012). ManR, a ClrB ortholog, participates in the XlnR-independent signaling pathway in Aspergillus oryzae and A. aculeatus (Ogawa et al. 2013; Tsumura et al. 2021). Although regulators involved in the regulation mechanisms of cellulolytic and xylanolytic enzyme genes have been identified, the details of these regulation mechanisms have not been clarified.

We previously established a positive screening method to identify regulators involved in the celluloseresponsive induction pathway in A. aculeatus by screening for 5-fluoroorotic acid (5-FOA)-resistant mutants on minimal medium (MM) with wheat brans as a sole carbon source (Kunitake et al. 2011; Kunitake et al. 2013). Using this method, we identified ClbR, dipeptidyl peptidase IV, and SepM as regulators stimulating the expression of cellulolytic enzyme genes in response to cellobiose (Kunitake et al. 2015; Kunitake et al. 2013; Tani et al. 2017; Tsumura et al. 2021). ClbR is a Zn(II) 2 Cys6-type transcriptional activator controlling the expression of cellulolytic enzyme genes in response to cellobiose (Kunitake, et al. 2013). SepM interacts with SepL, a putative kinase in the septation initiation network complex, which participates in septa formation and regulation mechanisms of the ManR-dependent signaling pathway (Tsumura et al. 2021). The molecular mechanisms of how the two different signaling 
pathways (the ManR- and XlnR-dependent signaling pathways), control gene expression in response to cellulose remain unknown.

To comprehensively understand the regulation mechanisms of cellulolytic enzyme genes, we further screened for a new regulator. In this study, we identified a putative protein kinase, SrpkF, which participates in the ManR- and XlnR-dependent signaling pathways to induce gene expression in response to cellulosic carbon sources.

\section{Materials and Methods}

Strains, transformation, marker recycling, and T-DNA insertion

All $A$. aculeatus strains used in this study were derived from wild-type $A$. aculeatus no. F-50 [NBRC 108789]. Unless otherwise stated, all strains were propagated at $30^{\circ} \mathrm{C}$ in an appropriately supplemented

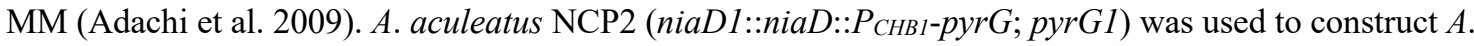
aculeatus strains for T-DNA insertion via Agrobacterium tumefaciens-mediated transformation.

Counterselection on 5-FOA and marker recycling were performed as described previously (Kunitake et al. 2011; Kunitake, et al. 2013). A. aculeatus MR12 (pyrG1; $\Delta k u 80$ ) was used for the disruption and complementation of the $\operatorname{srpkF}$ gene (Tani et al. 2013). Escherichia coli $\mathrm{DH} 5 \alpha \mathrm{F}^{\prime}$ was used for plasmid construction.

\section{Disruption and complementation of srpkF}

The $A$. aculeatus srpkF-deficient mutant ( $p y r G 1 ; \Delta k u 80 ; \Delta s r p k F$ ) was created by replacing $s r p k F$ with the A. nidulans orotidine 5 '-phosphate decarboxylase gene (AnpyrG) followed by marker recycling (Tani et al. 2013). To construct the $\operatorname{srpkF}$ deletion cassette, the $5^{\prime}$ and $3^{\prime}$ regions of $\operatorname{srpkF}$ (which play a key role in homologous recombination to replace $\operatorname{srpkF}$ with $A n p y r G$ ) were amplified from A. aculeatus genomic DNA using primer pairs 2.6k-5'srpkF/2.6k-5'srpkR and L-kinaseF/L-kinaseR, respectively. The AnpyrG gene was amplified from A. nidulans genomic DNA using the primer pair AnpyrG-KF/AnpyrG-KR. The 3 ' flanking region of sepM was amplified using the primer pair 2.6k-MsrpkF/M-kinaseR to eliminate Anpyr $G$ by intramolecular homologous recombination at the $\operatorname{srpkF}$ locus. The $5^{\prime}$ region of $A n p y r G$ (responsible for marker recycling) and the $3^{\prime}$ region were fused via PCR using the primer pair 2.6k5 'srpkF/L-kinaseR and subcloned into the EcoRV site of pBluescriptIIKS(+) to yield pDsrpkF. The srpkF deletion cassette was amplified via PCR using the primer pair 2.6k-5'srpkF/L-kinaseR from pDsrpkF and introduced into MR12 (pyrG1; $\Delta k u 80$ ) by the protoplast-PEG method to yield the A. aculeatus $\Delta s r p k F$ plus $p y r G$ strain (pyrG1; $\Delta k u 80 ; \Delta s r p k F:$ AnpyrG). Marker recycling was performed by spreading $1 \times 10^{4}$ transformant spores onto MM supplemented with $0.01 \%$ uridine and $1 \mathrm{mM} 5$-FOA. A. aculeatus $\Delta$ srpkF ( $\operatorname{pyrG1;} \Delta k u 80 ; \Delta s r p k F)$ was obtained following monospore isolation (Supplementary Fig. S1).

Supplementary Table S1 summarizes the primers used in this study. 
To complement $A$. aculeatus $\Delta s r p k F$, the srpkF promoter, open reading frame, and 3 '-untranslated region (UTR) were first amplified using the primer pair 2.6k-5'srpkF/M-kinaseR. AnpyrG and the 3' region required for homologous recombination at the $\operatorname{srpkF}$ locus were subsequently amplified with primer pairs AnpyrG-KF/AnpyrG-CR and C-kinaseF/C-kinaseR, respectively. The three DNA fragments were fused via PCR using the primer pair 2.6k-5'srpkF/C-kinaseR. Finally, A. aculeatus $\Delta s r p k F$ was transformed with the amplified DNA fragments to yield the $s r p k F$-complemented strain $(p y r G 1 ; \Delta k u 80$; $\Delta$ srpkF::srpkF::AnpyrG) (Supplementary Fig. S1).

The $A$. aculeatus SrpkF C-terminus deletion mutant was generated by introducing a stop codon at 1,129 nt. The ORF with 5' flanking region (-23-1,121 bp) and 3'-UTR of srpkF was amplified using primer pairs $\mathrm{pK}-\mathrm{F} / \mathrm{srpkFstopMR-R}$ and 3'UTRF/3'UTRR, respectively, and were fused via PCR using primer pair pK-F/3'UTRR. The region of AnpyrG for marker recycling and the $3^{\prime}$ region required for homologous recombination at the $\operatorname{srpkF}$ locus were subsequently amplified with primer pair MRstopsrpkF-F/L-kinaseR using pDsrpkF as a template. These fragments were fused via PCR using the primer pair pK-F/L-kinaseR. The fused fragments were introduced into MR12 (Supplementary Fig. S2). $A$. aculeatus $\Delta \operatorname{CsrpkF}$ (pyrG1; $\Delta k u 80 ; \Delta \operatorname{CsrpkF}_{1-327}$ ) strain was obtained after marker recycling. The $\Delta \mathrm{CsrpkF}$ strain was complemented by the same fragments used to complement $\Delta \operatorname{srpkF}$ (Supplementary Fig. S2).

The A. aculeatus srpkF-overexpressing strain (pyrG1::Ptef-srpkF::AapyrG; $\Delta k u 80 ; \Delta s r p k F)$ was generated by inserting an $s r p k F$-overexpressing cassette into the A. aculeatus pyrG locus in $\Delta s p r k F$. To introduce the expression cassette into the AapyrG locus, the AapyrG ORF with its promoter and terminator region, and the AapyrG 3' region were amplified using primer pairs AapyrGORFF/AapyrGORFR and AapyrG3'F/AapyrG3'R, respectively. The promoter region of the translation elongation factor $1 \alpha$ gene (Ptef) was amplified using primer set AaPtefF/AaPtefR and subsequently fused to the AapyrG 3' region via PCR using primer set AapyrG3'F/AaPtefR, followed by digestion with NotI and SacI. The amplified AapyrG ORF fragment was digested with KpnI and XbaI and subcloned into pBS KpnI and SalI sites. The plasmid was digested with NotI and SacI and ligated with the digested AapyrG 3' and Ptef fragments. The srpkF-overexpressing cassette was amplified using primer set 5'AapyrGF/3'AapyrGR. Finally, A. aculeatus $\Delta \operatorname{srpkF}$ was transformed with the amplified DNA fragments to yield the $\operatorname{srpkF-overexpressing~strain~(Supplementary~Fig.~S3).~}$

\section{Gene expression analysis by quantitative RT-PCR}

Quantitative RT-PCR (qRT-PCR) was used to quantify the expression of cellulase and hemicellulase genes on the basis of previous methods (Tani et al. 2017). In this study, 0.1\% Bacto ${ }^{\text {TM }}$ Tryptone (Thermo Fisher Scientific, Tokyo, Japan) was used as a neutral carbon source (noninducing condition). The indicated carbon sources were added to media supplemented with $0.1 \%$ Bacto $^{\mathrm{TM}}$ Tryptone to investigate the expression of test genes. Total RNA (500 ng) was used to amplify cDNA with ReverTra Ace ${ }^{\mathrm{TM}}$ qPCR 
RT-Master Mix (Toyobo, Tokyo, Japan). qRT-PCR was performed in a Thermal Cycler Dice ${ }^{\mathrm{TM}}$ RealTime System (Takara, Kyoto, Japan). For amplification, a SYBR® Green I assay using THUNDERBIRD ${ }^{\mathrm{TM}}$ SYBR ${ }^{\circledR}$ qPCR Mix (Toyobo) was performed in a $20 \mu 1$ reaction. The primers used for qRT-PCR are listed in Supplementary Table S1. Expression of the glyceraldehyde-3-phosphate dehydrogenase A gene $(g p d A)$ was used as an internal control. The specificity of the PCR amplification was confirmed by melting curve analysis. The expression profile of each gene was analyzed using a delta-delta CT method. More than three biological replicates were performed for each experiment, and each was tested in triplicate.

\section{Additional methods}

Genomic DNA preparation and Southern blot analysis were performed as described previously (Kunitake et al. 2013). An in-house $A$. aculeatus draft genome database was used to obtain the genomic sequence of the $\operatorname{srpkF}$ gene. Two independently amplified cDNA fragments were analyzed to determine the $s r p k F$ cDNA sequence. Conidia from the $A$. aculeatus strains were collected with $0.1 \%$ Tween ${ }^{\circledR} 80 / 0.8 \% \mathrm{NaCl}$ solution and counted with a hemocytometer. The number of conidia was normalized by colony area.

\section{Nucleotide sequence data}

The nucleotide sequence data from this study have been deposited in Japan's DNA Data Bank (DDBJ) Nucleotide Sequence Data Libraries. The accession number of $s r p k F$ in $A$. aculeatus is DDBJ Acc. no. LC638744.

\section{Results}

Isolation of a cellulose-responsive induction-deficient mutant from an A. aculeatus T-DNA inserted mutant library

We previously screened an A. aculeatus T-DNA inserted mutant library of approximately 12,000 transformants for strains that were 5-FOA-resistant and cellulose-responsive induction-deficient. We isolated five 5-FOA-resistant strains that showed reduced growth on media supplemented with 1\% Avicel ${ }^{\circledR}$ but normal growth on media supplemented with $1 \%$ glucose, $1 \%$ beechwood xylan, or $1 \%$ Dxylose (Tani et al. 2017). In the present study, we further analyzed one 5-FOA-resistant strain (Q3) that showed a strong correlation between the function of the disrupted gene (via T-DNA insertion) and the cellulose-responsive induction-deficient phenotype.

We first investigated $c b h I$ expression profiles in response to Avicel ${ }^{\circledR}$ in $\mathrm{Q} 3$ since the $c b h I$ promoter was fused to the pyrG gene and was used as bait to screen for factors involved in the cellulose-induced signaling pathway with the 5-FOA-resistant phenotype (Kunitake et al. 2013). Expression of $c b h I$ was induced in response to Avicel ${ }^{\circledR}$ at $9 \mathrm{~h}$ postinduction in the control strain (NCP2) but significantly reduced in Q3 to approximately 20\% of NCP2 expression levels ( $p<0.05$, Student's $t$-test) (Fig. 1). Expression of 
$x y n I b$ in response to Avicel ${ }^{\circledR}$ was also significantly reduced in Q3 to approximately $30 \%$ of $\mathrm{NCP} 2$ expression levels ( $p<0.05$, Student's $t$-test) (Fig. 1). These data suggest that the T-DNA insertion in Q3 disrupted a gene required for the induction of the test genes in response to Avicel ${ }^{\circledR}$.

\section{Serine-arginine protein kinase F participates in the early phase of cellulose induction}

The T-DNA integration pattern in Q3 was analyzed by Southern blot because approaches for the recovery of T-DNA flanking sequences by inverse PCR differ depending on the integration pattern. Genomic DNA isolated from Q3 was digested with EcoRI, XbaI and SpeI, HindIII, and PstI. Single digestion by EcoRI (unique site in the T-DNA left flanking region), HindIII (absent from the vector), and PstI (unique site in the vector backbone) and double digestion with $X b a \mathrm{I}$ and SpeI (unique site in the T-DNA right flanking region and absent from the vector, respectively) all yielded a single band (Fig. 2a). These data demonstrate that one copy of the T-DNA integrated into the Q3 genome. We attempted to amplify the TDNA flanking sequences via inverse PCR using Q3 genomic DNA digested with EcoRI and XbaI/SpeI to recover the right and left flanking sequences, respectively (Fig. 2b). We sequenced DNA fragments amplified via inverse PCR, which revealed that the T-DNA integrated into the ORF encoding the putative serine-threonine protein kinase (Fig. 2b). The right and left flanking sequences of the T-DNA were inserted at 1,122 and $1,137 \mathrm{nt}$ from the translation start site of the gene with a 15 bp deletion in the recipient genome. The putative protein kinase gene was composed of 1,443 bp with five exons and encoded a 416-amino acid protein. Based on a homology search in FungiDB

(https://fungidb.org/fungidb/), the putative protein kinase was most similar to ACLA_003920 in Aspergillus clavatus $(\mathrm{E}-\mathrm{value}=0.0$; Identities $=81 \%$ ). The gene was also similar to AN10082 in $\mathrm{A}$. nidulans, $\operatorname{SrpkF}$ (E-value $=7 \mathrm{e}-100$; Identities $=38 \%$ ), which is a member of a family of serine-threonine protein kinases that includes an expanded group of seven serine-arginine protein kinases (SRPK) in $A$. nidulans (de Souza et al. 2013). Therefore, this gene is designated as srpkF in A. aculeatus. The T-DNA insertion at $1,122 \mathrm{nt}$ from the translation start site of $\operatorname{srpkF}$ resulted in the production of a truncated protein of 327 amino acids of the original protein and seven additional amino acids (SNTDSLN) and a stop codon derived from the T-DNA fragment. These data suggest that $\operatorname{srpkF}$ is the candidate gene causing the cellulose-responsive induction-deficient phenotype of Q3.

\section{The srpkF gene expressed under the carbon starvation condition}

The expression profiles of $\operatorname{srpkF}$ were assessed under inducing (1\% Avicel ${ }^{\circledR}$ or $1 \% \mathrm{D}$-xylose), repressing (1\% D-glucose), carbon neutral ( $0.1 \%$ Bacto $^{\mathrm{TM}}$ Tryptone), and carbon starvation conditions of the cellulolytic and xylanolytic enzyme genes. Transcripts of $\operatorname{srpkF}$ were quantified using RNA prepared from the A. aculeatus wild-type strain grown under the following conditions: 1\% D-glucose medium for $3 \mathrm{~h}, 1 \%$ D-xylose medium for $3 \mathrm{~h}, 1 \%$ Avicel ${ }^{\circledR}$ medium for $9 \mathrm{~h}, 0.1 \%$ Bacto $^{\text {TM }}$ Tryptone medium for $3 \mathrm{~h}$, and no carbon source for $3 \mathrm{~h}$. The fold increases in $\operatorname{srpkF}$ gene expression compared with D-glucose 
medium were 2.3 (D-xylose), 44 (Avicel®), 59 (Bacto ${ }^{\text {TM }}$ Tryptone), and 98 (no carbon) (Fig. 3). $A$. aculeatus grew poorly on the $1 \%$ Avice ${ }^{\circledR}$ medium, $0.1 \%$ Bacto $^{\mathrm{TM}}$ Tryptone medium, and under the no carbon condition, whereas it grew well on $1 \%$ glucose and $1 \% \mathrm{D}$-xylose media, indicating that $A$. aculeatus could not acquire sufficient carbon from $1 \%$ Avicel ${ }^{\circledR}$ and $0.1 \%$ Bacto $^{\mathrm{TM}}$ Tryptone media. These data demonstrate that $\operatorname{srpkF}$ is expressed under conditions in which the carbon source is limited.

\section{Functional analysis of SrpkF in A. aculeatus}

To genetically analyze the function of $\operatorname{srpkF}$, the whole $\operatorname{srpkF}$ gene was deleted by homologous recombination $\left(\Delta s r p k F, p y r G^{+}\right)$followed by marker recycling to yield an A. aculeatus srpkF deletion mutant $\left(\Delta s r p k F, p y r G^{-}\right)$. Since the $\operatorname{srpkF}$ deletion mutant had never been isolated using the DNA fragment corresponding to the $1522 \mathrm{nt}$ region upstream from the translation start site that was used for homologous recombination, the DNA fragment from 1,523 to 2,613 nt upstream was used for targeting. Furthermore, the $3^{\prime}$ flanking region of $\operatorname{srpkF}$ was deleted after marker recycling in $\triangle s r p k F$. Thus, the generated $\operatorname{srpkF}$ mutant deleted $\operatorname{srpkF}$ with $1.5 \mathrm{~kb}$ upstream and $1.3 \mathrm{~kb}$ downstream flanking sequences. $\Delta \operatorname{srpkF}$ was transformed with $\operatorname{srpkF}$ DNA fragment yielding a complemented strain $\left(\operatorname{srpkF} F^{+}\right)$ (Supplementary Fig. S1). To generate a C-terminal deletion mutant of SrpkF composed of 327 amino acids $(\triangle \mathrm{CsrpkF})$ in $\mathrm{Q} 3$, a stop codon was introduced at 1,129 nt from the translation start site of the ORF followed by marker recycling of $p y r G$ to yield $\Delta \operatorname{CsrpkF}\left(\operatorname{SrpkF}_{1-327}, p y r G^{-}\right) . \Delta \operatorname{CsrpkF}$ was also complemented by the $\operatorname{srpkF}$ DNA fragment. We confirmed that gene replacement and complementation occurred as expected by Southern blot analysis (Supplementary Fig. S2). MR12, $\Delta \operatorname{srpkF,~} \Delta$ CsrpkF, and $\operatorname{srpkF^{+}}$ grew equally well on MM supplemented with $1 \%$ D-glucose, $1 \%$ D-xylose, and $1 \%$ Avicel® (data not shown). Since the expression profiles of test genes in the complemented $\Delta$ CsrpkF strain were no different from those in MR12 and $\operatorname{srpkF^{+}}$, we used $\operatorname{srpkF^{+}}$ as a control strain in this study (data not shown).

We investigated the effect of $\operatorname{srpkF}$ deletion on gene expression in response to cellulosic carbon sources. Transcripts of $c b h I$ and $x y n I b$ were quantified at 6 and $9 \mathrm{~h}$ postinduction in the MR12, $\Delta \mathrm{Cs} r p k F$, $\Delta s r p k F$, and $\operatorname{srpkF} F^{+}$strains. The expression of $c b h I$ and $x y n I b$ was reduced in $\Delta$ CsrpkF and $\Delta s r p k F$ only under inducing conditions. The fold induction of $c b h I$ in response to Avicel® decreased significantly compared with MR12 at $9 \mathrm{~h}$ postinduction to $50 \%$ in $\triangle \operatorname{srpkF}$ and $31 \%$ in $\Delta \operatorname{CsrpkF}(p<0.05$, Student's $t$ test). Similarly, the fold induction of xynIb significantly decreased to $26 \%$ in $\Delta s r p k F$ and $32 \%$ in $\Delta \operatorname{CsrpkF}(p<0.05$, Student's $t$-test) (Supplementary Fig. S4 and Fig. 4). These data demonstrated that SrpkF is involved in the expression of both genes in response to Avicel®. Since their expression levels decreased similarly in both $\triangle \mathrm{CsrpkF}$ and $\Delta s r p k F$ and were restored by srpkF complementation, we further analyzed the SrpkF function using only $\Delta s r p k F$. Expression of $c e l 7 b$ significantly decreased to $55 \%$ at $9 \mathrm{~h}$ postinduction in $\Delta \operatorname{srpkF}$ compared with MR12 ( $p<0.05$, Student's $t$-test), which was restored 
in $s r p k F^{+}$(Fig. 4). The expression of $c m c 2, c m c 1$, and $x y n I a$, decreased to approximately $60 \%$, but this reduction was not statistically significant (Fig. 4).

\section{Overexpression of $\operatorname{srpkF}$}

To assess the effect of overexpression of $\operatorname{srpkF}$ on the expression of genes encoding cellulosic biomassdegrading enzymes in A. aculeatus, we constructed an $s r p k F$-overexpressing strain that constitutively expresses $\operatorname{srpkF}$ under the control of the translation elongation factor $1 \alpha$ gene promoter (Ptef), a highlevel constitutive promoter in Aspergillus (Kunitake et al. 2015). The srpkF-overexpressing cassette was introduced into the $p y r G$ locus in $\triangle s r p k F$ as a single copy by homologous recombination, as confirmed by Southern blot analysis (Supplementary Fig. S3), yielding the $s r p k F$-overexpressing strain (OEsrpkF). The expression of $s r p k F$ increased four to seven times in the presence of cellobiose with 1-deoxynojirimycin (DNJ) and approximately nine times in the presence of D-xylose (Supplementary Fig. S5). Since $A$. aculeatus produces $\beta$-glucosidase, which effectively hydrolyzes cellobiose to glucose, DNJ was added as a $\beta$-glucosidase inhibitor (Tani et al. 2012). The physiological phenotype of the OEsrpkF strain was no different from MR12, $\triangle \mathrm{CsrpkF}$, and $\Delta \operatorname{srpkF}$ (data not shown).

We assessed the effect of $\operatorname{srpkF}$ overexpression on the expression of cellulosic biomass-degrading enzyme genes in response to physiological inducers. The expression of $c b h I, c m c 2$, and $c e l 7 b$ is induced in response to cellobiose by the ManR-dependent signaling pathway. Under the inducing condition, the expression of $c b h I, c m c 2$, and cel7b generally increased in OEsrpkF compared with MR12 (Fig. 5 a and b); however, their expression profiles varied. The expression of $c h b I$ was induced at 2, 3, and $4 \mathrm{~h}$ postinduction in OEsrpkF only under the inducing condition, resulted in a significant increase of the fold induction (Fig. $5 \mathrm{a}$ and b). By contrast, the overexpression of $\operatorname{srpkF}$ did not significantly increase either the expression level or fold induction of $c m c 2$ (Fig. $5 \mathrm{a}$ and b). The expression of $c e l 7 b$ significantly stimulated under both inducing and noninducing conditions such that there was no significant difference in the fold induction, indicating that SprkF stimulated the expression of cel7b at a basal level (Fig. 5 a and b).

The expression of $x y n I b$ and $c m c l$ is induced in response to D-xylose by the XlnR-dependent signaling pathway. The expression of $x y n I b$ and $c m c l$ was enhanced markedly at 1.5 and $3.0 \mathrm{~h}$ postinduction in OEsrpkF under the D-xylose-inducing condition (Fig. $5 \mathrm{c}$ and d). These data confirm that SrpkF participates in not only the cellulose-responsive signaling pathway but also in the xyloseresponsive signaling pathway.

\section{Discussion}

In this study, we demonstrated that deletion of $\operatorname{srpkF}$ resulted in the reduction of cellulase and xylanase gene expression in response to cellulosic carbon sources. Since the overexpression of $s r p k F$ promoted the 
gene expression in the early induction stages in response to cellobiose and D-xylose, we confirmed that SrpkF in A. aculeatus is a positive regulator in response to cellulosic and xylanolytic carbon sources.

A comparison of amino acid sequences using the FASTA algorithm revealed that srpkF orthologs are highly conserved in the Aspergillus section Nigri. Orthologs are also present in a few strains from other sections and other genera, such as $A$. nidulans (AN10082, E-value $=2 \mathrm{e}-91$; Identities $=42 \%$ ), Penicillium rubens $(\operatorname{Pc} 12 \mathrm{~g} 16110, \mathrm{E}-\mathrm{value}=1 \mathrm{e}-104 ;$ Identities $=47 \%)$, and Coccidioides immitis $(\mathrm{CIMG}$-04484, Evalue $=6 \mathrm{e}-103$; Identities $=44 \%$ ) (Fig. 6). AN10082 in A. nidulans encodes a serine-arginine protein kinase F (SrpkF) for which a deletion mutant did not show a distinguishing phenotype (de Souza et al. 2013). SRPK was first identified as a cell cycle-regulated kinase specific for SR proteins, which are a family of pre-mRNA splicing factors containing SR domains that consist largely of serine/arginine repeats (Gui et al. 1994b; Gui et al. 1994c). Members of this kinase family are known to phosphorylate serines within the SR domain and are widely conserved in eukaryotes. Although S. cerevisiae encodes a single SRPK family member, Sky1, A. nidulans and A. aculeatus encode seven SRPKs from SrpkA to G (de Souza et al. 2013) and four SRPKs, respectively. SrpkA proteins in Aspergillus are highly conserved with $S$. cerevisiae Sky1, suggesting that they modulate the subcellular localization and function of SerArg rich splicing-factor proteins (Dagher and Fu 2001; Gui et al. 1994a). However, other SRPKs possess various domains, suggesting functional diversification. Few nonsplicing functions of SRPKs have been reported (Gou et al. 2020; Hong et al. 2012; Wang et al. 2017), and it remains unclear whether SRPKs have evolved further regulatory roles (Bustos et al. 2020). The functions of SRPKs might be illuminated by investigating their gene expression profiles since some genes exhibit highly tissue-specific profiles (Nakagawa et al. 2005; Wang et al. 1998).

The expression of $s r p k F$ increased under carbon-limited conditions, such as the no carbon and Avicel ${ }^{\circledR}$ conditions (Fig. 3), which showed a strong correlation between SrpkF function in cellulose-responsive induction and expression profiles. To narrow the possible pathways involving SrpkF, we focused on two signaling pathways involved in cellulose-responsive induction in A. aculeatus. One is the ManRdependent signaling pathway that induces expression of $c b h I, c m c 2$, and $c e l 7 b$. Contrary to our assumptions, the effects of deletion and overexpression of $\operatorname{srpkF}$ were not consistent among the three genes. Complex regulations were observed in our previous study as well. The overexpression of $c l b R$, a putative transcription factor involved in cellobiose-responsive induction in A. aculeatus, did not affect the expression of $c b h I$ and $c m c 2$ but reduced the expression of $c e l 7 b$; nevertheless, the deletion of $c l b R$ reduced their expression levels in response to Avicel® (Kunitake et al. 2015). These data demonstrate that various factors are involved in the ManR-dependent signaling pathway in response to cellulosic carbon sources in A. aculeatus.

Interestingly, the overexpression of $\operatorname{srpkF}$ promoted xylanase gene expression in response to D-xylose, whereas no effect of the $\operatorname{srpkF}$ deletion was observed on the xylose-responsive expression of the xylanase gene, indicating that the function of SrpkF is critically regulated at the transcription level. Note that $x y n I b$ 
and $c m c l$ are under the control of $X \operatorname{lnR}$ (Tani et al. 2012). XlnR is constitutively expressed in Aspergillus oryzae and is phosphorylated in the presence of D-xylose while rapidly dephosphorylated by removing Dxylose from the media (Noguchi et al. 2011). Although it is unknown if ManR is phosphorylated, SrpkF is a candidate kinase to phosphorylate factors involved in the ManR-and XlnR-dependent signaling pathways.

Various protein kinases are involved in establishing a complex regulatory network to maintain the precise balance of carbon sources required for growth and hydrolytic enzyme production. Trichoderma mitogen-activated protein kinase, Tmk2, is involved in cell wall integrity, sporulation, and cellulase production (Wang et al. 2014). The cyclic AMP-dependent protein kinase A (PKA) affects cellulase gene expression in response to light in T. reesei (Schuster et al. 2012). PKA in A. nidulans is also involved in the repression of cellulolytic enzyme genes in response to carbon sources (Kunitake et al. 2019). However, the molecular mechanisms of how their kinases control the expression of cellulolytic enzyme genes remain unknown.

A recent study revealed the levels of conservation and diversity in the regulatory mechanisms of cellulolytic enzyme genes in Ascomycete fungi (Kunitake and Kobayashi 2017). XlnR mainly regulates the expression of xylanolytic enzyme genes and is only slightly involved in the expression of cellulolytic enzyme genes in response to cellulose in Aspergillus oryzae and A. aculeatus (Marui et al. 2002; Tani et al. 2012). By contrast, $\mathrm{Xyr} 1$, an $\mathrm{X} \operatorname{lnR}$ ortholog in T. reesei, is a master regulator governing the expression of xylanolytic and cellulolytic enzyme genes in response to various carbon sources such as D-xylose, sophorose, galactose, and lactose (Stricker et al. 2006; Stricker et al. 2007). XLR-1 in N. crassa is involved in the induction of xylanolytic enzyme genes but not significantly involved in the expression of cellulolytic enzyme genes (Sun et al. 2012). The expression of cellulolytic enzyme genes is mainly regulated by ManR in A. oryzae and A. aculeatus (Ogawa et al. 2013; Tsumura et al. 2021) and its orthologs ClrB in A. nidulans and CLR-2 in N. crassa (Coradetti et al. 2012; Coradetti et al. 2013). Orthologous genes of $\operatorname{srpkF}$ were absent from the genomes of genera of cellulase-producing fungi, such as Trichoderma and Neurospora species. Since the acquisition of paralogous genes establishes new signal transduction pathways (Baker et al. 2013), the acquisition of srpkF in Aspergillus might lead to a different regulation mechanism of cellulolytic enzyme genes. The logical next step is to identify the target protein of SrpkF, which will help to understand the complex regulatory mechanisms of cellulolytic enzyme genes in Aspergillus.

\section{References}


Adachi H, Tani S, Kanamasa S, Sumitani J, Kawaguchi T (2009) Development of a homologous transformation system for Aspergillus aculeatus based on the $s C$ gene encoding ATPsulfurylase. Biosci Biotechnol Biochem 73:1197-1199. doi: 10.1271/bbb.80772

Baba Y, Sumitani J, Tani S, Kawaguchi T (2015) Characterization of Aspergillus aculeatus $\beta$-glucosidase 1 accelerating cellulose hydrolysis with Trichoderma cellulase system. AMB Express 5:3. doi: 10.1186/s13568-014-0090-3

Baker CR, Hanson-Smith V, Johnson AD (2013) Following gene duplication, paralog interference constrains transcriptional circuit evolution. Science 342:104-108. doi: 10.1126/science.1240810

Bischof RH, Ramoni J, Seiboth B (2016) Cellulases and beyond: the first 70 years of the enzyme producer Trichoderma reesei. Microb Cell Fact 15:106. doi: 10.1186/s12934-016-0507-6

Bustos F, Segarra-Fas A, Nardocci G, Cassidy A, Antico O, Davidson L, Brandenburg L, Macartney TJ, Toth R, Hastie CJ, Moran J, Gourlay R, Varghese J, Soares RF, Montecino M, Findlay GM (2020) Functional diversification of SRSF protein kinase to control ubiquitin-dependent neurodevelopmental Signaling. Dev Cell 55:629-647. e627 doi: 10.1016/j.devcel.2020.09.025

Coradetti S, Craig J, Xiong Y, Shock T, Tian C, Glass N (2012) Conserved and essential transcription factors for cellulase gene expression in Ascomycete fungi. Proc Natl Acad Sci USA 109:73977402. doi: 10.1073/pnas.1200785109

Coradetti ST, Xiong Y, Glass NL (2013) Analysis of a conserved cellulase transcriptional regulator reveals inducer-independent production of cellulolytic enzymes in Neurospora crassa. MicrobiologyOpen 2:595-609. doi: 10.1002/mbo3.94

Dagher SF, Fu XD (2001) Evidence for a role of Skylp-mediated phosphorylation in 3' splice site recognition involving both Prp8 and Prp17/Slu4. RNA 7:1284-1297. doi:

$10.1017 / \mathrm{s} 1355838201016077$

de Souza CP, Hashmi SB, Osmani AH, Andrews P, Ringelberg CS, Dunlap JC, Osmani SA (2013) Functional analysis of the Aspergillus nidulans kinome. PLoS One 8:e58008. doi: 10.1371/journal.pone.0058008

Gui JF, Chandler SD, Fu XD (1994a) Purification and characterization of a kinase specific for the serineand arginine-rich pre-mRNA splicing factors. Proc Natl Acad Sci U S A 91: 10824-10828 doi: 10.1073/pnas.91.23.10824

Gui JF, Lane WS, Fu XD (1994b) A serine kinase regulates intracellular localization of splicing factors in the cell cycle. Nature 1994:678-682. doi: 10.1038/369678a0

Gui JF, Tronchère H, Chandler SD, Fu XD (1994c) Purification and characterization of a kinase specific for the serine- and arginine-rich pre-mRNA splicing factors. Proc Natl Acad Sci U S A 91:10824-10828. doi: 10.1073/pnas.91.23.10824

Kunitake E, Kawamura A, Tani S, Takenaka S, Ogasawara W, Sumitani J, Kawaguchi T (2015) Effects of $c l b R$ overexpression on enzyme production in Aspergillus aculeatus vary depending on the 
cellulosic biomass-degrading enzyme species. Biosci Biotechnol Biochem 79:488-495. doi: $10.1080 / 09168451.2014 .982501$

Kunitake E, Kobayashi T (2017) Conservation and diversity of the regulators of cellulolytic enzyme genes in Ascomycete fungi. Curr Genet 10.1007/s00294-017-0695-6

Kunitake E, Li Y, Uchida R, Nohara T, Asano K, Hattori A, Kimura T, Kanamaru K, Kimura M, Kobayashi T (2019) CreA-independent carbon catabolite repression of cellulase genes by trimeric G-protein and protein kinase A in Aspergillus nidulans. Curr Genet 65:941-952. doi: 10.1007/s00294-019-00944-4

Kunitake E, Tani S, Sumitani J, Kawaguchi T (2011) Agrobacterium tumefaciens-mediated transformation of Aspergillus aculeatus for insertional mutagenesis. AMB Express 1:46. doi: 10.1186/2191-0855-1-46

Kunitake E, Tani S, Sumitani J, Kawaguchi T (2013) A novel transcriptional regulator, ClbR, controls the cellobiose- and cellulose-responsive induction of cellulase and xylanase genes regulated by two distinct signaling pathways in Aspergillus aculeatus. Appl Microbiol Biotechnol 97:2017-2028. doi: 10.1007/s00253-012-4305-8

Marui J, Kitamoto N, Kato M, Kobayashi T, Tsukagoshi N (2002) Transcriptional activator, AoXlnR, mediates cellulose-inductive expression of the xylanolytic and cellulolytic genes in Aspergillus oryzae. FEBS Lett 528:279-282. doi: 10.1016/s0014-5793(02)03328-8

Nakagawa O, Arnold M, Nakagawa M, Hamada H, Shelton JM, Kusano H, Harris TM, Childs G, Campbell KP, Richardson JA, Nishino I, Olson EN (2005) Centronuclear myopathy in mice lacking a novel muscle-specific protein kinase transcriptionally regulated by MEF2. Genes Dev 19:2066-2077. doi: 10.1101/gad.1338705

Nakazawa H, Kawai T, Ida N, Shida Y, Kobayashi Y, Okada H, Tani S, Sumitani J, Kawaguchi T, Morikawa Y, Ogasawara W (2012) Construction of a recombinant Trichoderma reesei strain expressing Aspergillus aculeatus $\beta$-glucosidase 1 for efficient biomass conversion. Biotech Bioeng 109:92-99. doi: 10.1002/bit.23296

Noguchi Y, Tanaka H, Kanamaru K, Kato M, Kobayashi T (2011) Xylose triggers reversible phosphorylation of $\mathrm{X} \operatorname{lnR}$, the fungal transcriptional activator of xylanolytic and cellulolytic genes in Aspergillus oryzae. Biosci Biotechnol Biochem 75:953-959. doi: 10.1271/bbb.100923

Ogawa M, Kobayashi T, Koyama Y (2013) ManR, a transcriptional regulator of the $\beta$-mannan utilization system, controls the cellulose utilization system in Aspergillus oryzae. Biosci Biotechnol Biochem 77:426-429. doi: 10.1271/bbb.120795

Payne CM, Knott BC, Mayes HB, Hansson H, Himmel ME, Sandgren M, Ståhlberg J, Beckham GT (2015) Fungal cellulases. Chem Rev 115:1308-1448. doi: 10.1021/cr500351c 
Schuster A, Tisch D, Seidl-Seiboth V, Kubicek CP, Schmoll M (2012) Roles of protein kinase a and adenylate cyclase in light-modulated cellulase regulation in Trichoderma reesei. Appl Environ Microbiol 78:2168-2178. doi: 10.1128/AEM.06959-11

Stricker AR, Grosstessner-Hain K, Wurleitner E, Mach RL (2006) Xyr1 (xylanase regulator 1) regulates both the hydrolytic enzyme system and D-xylose metabolism in Hypocrea jecorina. Eukaryot Cell 5:2128-2137. doi: 10.1128/ec.00211-06

Stricker AR, Steiger MG, Mach RL (2007) Xyr1 receives the lactose induction signal and regulates lactose metabolism in Hypocrea jecorina. FEBS Lett 581:3915-3920. doi: 10.1016/j.febslet.2007.07.025

Sun J, Tian C, Diamond S, Glass NL (2012) Deciphering transcriptional regulatory mechanisms associated with hemicellulose degradation in Neurospora crassa. Eukaryot Cell 11:482-493. doi: 10.1128/EC.05327-11

Tani S, Kanamasa S, Sumitani J, Arai M, Kawaguchi T (2012) XlnR-independent signaling pathway regulates both cellulase and xylanase genes in response to cellobiose in Aspergillus aculeatus. Curr Genet 58:93-104. doi: 10.1007/s00294-012-0367-5

Tani S, Kawaguchi T, Kobayashi T (2014) Complex regulation of hydrolytic enzyme genes for cellulosic biomass degradation in filamentous fungi. Appl Microbiol Biotechnol 98:4829-4837. doi: 10.1007/s00253-014-5707-6

Tani S, Tsuji A, Kunitake E, Sumitani J, Kawaguchi T (2013) Reversible impairment of the ku80 gene by a recyclable marker in Aspergillus aculeatus. AMB Express 3:4. doi: 10.1186/2191-0855-3-4

Tani S, Yuki S, Kunitake E, Sumitani J, Kawaguchi T (2017) Dipeptidyl peptidase IV is involved in the cellulose-responsive induction of cellulose biomass-degrading enzyme genes in Aspergillus aculeatus. Biosci Biotechnol Biochem 81:1227-1234. doi: 10.1080/09168451.2017.1295800

Tsumura R, Sawada K, Kunitake E, Sumitani J, Kawaguchi T, Tani S (2021) A component of the septation initiation network complex, AaSepM, is involved in multiple cellulose-responsive signaling pathways in Aspergillus aculeatus. Appl Microbiol Biotechnol 105:1535-1546. doi: 10.1007/s00253-021-11110-7

van Peij N, Visser J, de Graaff L (1998) Isolation and analysis of XlnR, encoding a transcriptional activator co-ordinating xylanolytic expression in Aspergillus niger. Mol Microbiol 27:131-142. doi: 10.1046/j.1365-2958.1998.00666.x

Wang HY, Lin W, Dyck JA, Yeakley JM, Songyang Z, Cantley LC, Fu XD (1998) SRPK2: A differentially expressed SR protein-specific kinase involved in mediating the interaction and localization of pre-mRNA splicing factors in mammalian cells. J Cell Biol 140:737-750. doi: 10.1083/jcb.140.4.737 
Wang M, Dong Y, Zhao Q, Wang F, Liu K, Jiang B, Fang X (2014) Identification of the role of a MAP kinase Tmk2 in Hypocrea jecorina (Trichoderma reesei). Sci Rep 4:6732. doi:

$10.1038 /$ srep06732 


\section{Supplementary Table S1}

\begin{tabular}{|c|c|}
\hline Name & Sequence $\left(5^{\prime}\right.$ to $\left.3^{\prime}\right)$ \\
\hline \multicolumn{2}{|l|}{ For srpkF deletion } \\
\hline $2.6 \mathrm{k}-5^{\prime} \mathrm{srpkF}$ & TACTGCCTTGGTCGTGACAATCG \\
\hline $2.6 \mathrm{k}-5^{\prime} \mathrm{srpkR}$ & CCGATGACAAAGTACGTAGTCCGCGAAACC \\
\hline 2.6k-MsrpkF & CTACGTACTTTGTCATCGGATTCAGTCAAGC \\
\hline M-kinaseR & TCTGCGGCAAGCCAGAGCCACTTAACTG \\
\hline AnpyrG-KF & CTCTGGCTTGCCGCAGACAATGCTCTC \\
\hline AnpyrG-KR & GTCCTTGCCATATCGTTCAGAGCTGGTCAC \\
\hline L-kinaseF & AACGATATGGCAAGGACCTAATCTGATCG \\
\hline L-kinaseR & GAGTATGGTGGAGGCTAGATTG \\
\hline \multicolumn{2}{|c|}{ For srpkF and $\triangle$ CsrpkF complementation } \\
\hline $2.6 \mathrm{k}-5^{\prime} \mathrm{srpkF}$ & TACTGCCTTGGTCGTGACAATCG \\
\hline C2.6k-5'srpkR & TTGCGACCAAAGTACGTAGTCCGCGAAACC \\
\hline C4k-srpkF & CTACGTACTTTGGTCGCAAGACTGAAACTG \\
\hline M-kinase-R & TCTGCGGCAAGCCAGAGCCACTTAACTG \\
\hline AnpyrG-KF & CTCTGGCTTGCCGCAGACAATGCTCTC \\
\hline AnpyrG-CR & AGACTGTAAACATATCGTTCAGAGCTGGTCAC \\
\hline C-kinaseF & GAACGATATGTTTACAGTCTTGGCCTTCCC \\
\hline C-kinaseR & CAGGACAACGAAAGCATGAG \\
\hline \multicolumn{2}{|l|}{ For $\triangle C s r p k F$} \\
\hline $\mathrm{pK}-\mathrm{F}$ & GATTTCCCCACACATCAACC \\
\hline srpkFstopMR-R & CCAAGGATCTCAATTAGAGAACGCGGAGGTATC \\
\hline 3'UTRF & CGTTCTCTAATTGAGATCCTTGGTTCCAGGAATAG \\
\hline 3'UTRR & CCGATGACAATTGTGGTGGATTTGCAGA \\
\hline MRstop-srpkF-F & CCACCACAATTGTCATCGGATTCAGTCAAGC \\
\hline L-kinaseR & GAGTATGGTGGAGGCTAGATTG \\
\hline \multicolumn{2}{|l|}{ For Ptef plasmid } \\
\hline AapyrGORFF & AAGAGCTCTTGTATGCGACTGGGCTTTC \\
\hline AapyrGORFR & GGTACTGTACCGCTTGCAGGATGTTGTTTAG \\
\hline AaPtefF & CCTGCAAGCGGTACAGTACCTACGTGCTGCAATC \\
\hline AaPtefR & AATGCGGCCGCGGTGACGGTGTTGTGAAGTG \\
\hline AapyrG3'F & ATACTCGAGCGGATTCCAGATCTGGAGG \\
\hline AapyrG3'R & AAGGTACCCCTGTTCTGGTTGG \\
\hline \multicolumn{2}{|c|}{ For srpkF overexpression } \\
\hline OE-srpkF & TAAGCGGCCGCATGACGGCACTATTGAGCTCG \\
\hline
\end{tabular}




\begin{tabular}{ll} 
OE-srpkR & ATTGTGGTGGATTTGCAGACC \\
5'AapyrGF & AAGGTACCGCGTCGCGAAGGAGTTGT \\
3'AapyrGR & AAGAGCTCTTGTATGCGACTGGGCTTTC \\
\hline For qRT-PCR & \\
QgpdA-F & CTCCTCCACTGGTGCTGCT \\
QgpdA-R & GACTTCTCGAGACGGCAGG \\
QcbhI-F & ACCATGTGCACAGGCGATAC \\
QcbhI-R & TGCTGTTGTCGACGGTCTTT \\
Qcel7b-F & ACCAGGGACTCACCAAGATG \\
Qcel7b-R & GGCGTTGGTGGGGTAG \\
Qcmc1-F & TGGCTCGCAGAAGACCTACA \\
Qcmc1-R & ACCAGTTCGAGACGGAGAGC \\
Qcmc2-F & CCCTGCTGCAGAGCTATGTG \\
Qcmc2-R & GGCAGTGGAGGTGGAGGTAG \\
QxynIb-F & GAACTTCGTCGGTGGAAAGG \\
QxynIb-R & CCAGGGTTGTACTCCCCGTA \\
QsrpkFF & CCTGAATTAATTCCAGAGAGC \\
QsrpkFR & TGAGTAAATCTTCCATGCTG \\
\hline \hline
\end{tabular}




\section{Figure Legends}

Fig. 1. Identification of the cellulose-responsive induction-deficient mutant. qRT-PCR analysis of $c b h I$ and $x y n I b$ expression at $9 \mathrm{~h}$ postinduction with $1 \%(\mathrm{w} / \mathrm{v})$ Avicel ${ }^{\circledR}$ was performed for control (NCP2) and Q3 strains. The relative expression corresponds to the ratio of the mean expression levels of $c b h I$ divided by those of $g p d A$, the reference gene. The relative expression levels are the means of three independent experiments, and the error bars indicate the standard deviations. Letters indicate significant differences between groups $(p<0.05$, Student's $t$-test).

Fig. 2. Determination of T-DNA integration pattern and identification of the disrupted gene. Deduced TDNA integration pattern via Southern blotting analysis (a) and a schematic representation of the SrpkF $\operatorname{locus}(\mathrm{b})$.

Fig. 3. qRT-PCR analysis of the srpkF expression in wild-type $A$. aculeatus under the following conditions: 1\% D-glucose for $3 \mathrm{~h}(\mathrm{G}), 1 \% \mathrm{D}$-xylose for $3 \mathrm{~h}(\mathrm{X}), 1 \%$ Avicel ${ }^{\circledR}$ for $9 \mathrm{~h}(\mathrm{~A}), 0.1 \%$ Bacto $^{\mathrm{TM}}$ Tryptone for $3 \mathrm{~h}(\mathrm{~B})$, and no carbon source for $3 \mathrm{~h}(\mathrm{~N})$. The relative expression corresponds to the ratio of the mean expression levels of $\operatorname{srpkF}$ divided by those of $g p d A$. The relative expression levels are the means of three independent experiments, and the error bars indicate the standard deviations. Letters indicate significant differences between groups ( $p<0.05$, one-way ANOVA).

Fig. 4. Effect of $s r p k F$ deletion on expression of cellulase and hemicellulase genes. qRT-PCR results of each gene in MR12 (M, black bars), $\Delta \operatorname{srpkF}\left(\Delta\right.$, white bars), and $\operatorname{srpkF^{+}}$ (+, gray bars) incubated for 6 and $9 \mathrm{~h}$ under the noninduced condition (-) and the $1 \%$ Avicel ${ }^{\circledR}$-inducing condition (Avi). The relative expression corresponds to the ratio of the mean expression levels of each gene divided by that of $g p d A$. The relative expression levels are the means of three independent experiments, and the error bars indicate the standard deviations. Letters indicate significant differences between groups $(p<0.05$, one-way ANOVA)

Fig. 5. Effect of $\operatorname{srpkF}$ overexpression on expression of cellulase and hemicellulase genes. qRT-PCR results of each gene in MR12 (M, black bars) and the $\operatorname{srpkF-overexpressing~strain~OEsrpkF~(O,~striped~}$ bars). a, b: RNA was prepared from strains grown for $2-4 \mathrm{~h}$ in the presence of $0.1 \%$ cellobiose with 50 $\mu \mathrm{g} / \mathrm{L}$ DNJ. The expression level of each gene was normalized by that of the gpdA (a). The fold induction of each test gene reflects the gene expression level under the inducing condition divided by that under the noninducing condition (b). c, d: RNA was prepared from strains grown for 1.5-3.0 h in the presence of $1 \%(\mathrm{w} / \mathrm{v})$ D-xylose. The expression level of each gene was normalized by that of the $g p d A(\mathrm{c})$. d. The fold induction of each test gene reflects the gene expression level under the inducing condition divided by that under the noninducing condition (d). For all panels, the results shown are the means of three 
independent experiments, and the error bars indicate the standard deviations. An asterisk indicates a significant difference between the expression of test genes in MR12 and in OEsrpkF $(p<0.05$, Student's $t$-test).

Fig. 6. Phylogenetic analysis of serine-arginine protein kinase-like genes. The phylogenetic relationship of $s r p k$ orthologs is shown as a consensus neighbor-joining tree based on sequences orthologous to $s r p k F$ in $A$. aculeatus. The alignment was performed using ClustalW. The individual nodes of the resulting trees were examined with 1000 bootstrap replicates; only values below 1000 are shown.

Supplementary Fig. S1. Construction of the $\Delta \operatorname{srpkF}$ and $\operatorname{srpkF^{+}}$ strains. The upper portion of the figure shows restriction enzyme maps of the $\operatorname{srpkF}$ loci in the MR12, $\Delta \operatorname{srpkF}$, and $\operatorname{srpkF} F^{+}$strains. The underlined DNA regions were used as DNA probes for Southern blot analysis to detect DNA fragments digested with BamHI (lower portion of figure).

Supplementary Fig. S2. Construction of the $\triangle \mathrm{CsrpkF}$ and complement strains $\left(\mathrm{Csrpk} F^{+}\right)$. The upper portion of the figure shows restriction enzyme maps of the $\operatorname{srpkF}$ loci in the MR12, $\triangle \mathrm{CsrpkF}$, and complement strains. An asterisk indicates the position of a stop codon. The underlined DNA regions were used as DNA probes for Southern blot analysis to detect DNA fragments digested with DraI (lower portion of figure).

Supplementary Fig. S3. Construction of the OEsrpkF strain. The upper portion of the figure shows restriction enzyme maps of the $\operatorname{srpkF}$ loci in the MR12 and OEsrpkF strains. The underlined DNA regions were used as DNA probes for Southern blot analysis to detect DNA fragments digested with PstI (lower portion of figure).

Supplementary Fig. S4. Effect of $s r p k F$ deletion on expression of $c b h I$ and $x y n I b$. qRT-PCR results of each gene in MR12 (M), $\Delta \operatorname{srpkF}(\Delta), \operatorname{srpk} F^{+}(+), \Delta \operatorname{Csprk} F(\Delta \mathrm{C})$, and the complement strain of $\Delta \operatorname{Csprk} F$ $(\mathrm{C}+)$ incubated for $9 \mathrm{~h}$ under the noninduced condition and the $1 \%$ Avicel $®$-inducing condition The expression level of each gene was normalized by that of the gpdA. The fold induction of each test gene (the gene expression level under the inducing condition divided by that under the noninducing condition) is the mean of three independent experiments, and the error bars indicate the standard deviations. Letters indicate significant differences between groups ( $p<0.05$, one-way ANOVA).

Supplementary Fig. S5. Expression of $s r p k F$ in the overexpression strain. qRT-PCR analysis of $s r p k F$ in MR12 (M) and the $s r p k F$-overexpressing strain (OEsrpkF, OE). RNA was prepared from strains grown from $24 \mathrm{~h}$ in the presence of $0.1 \%$ cellobiose with $50 \mu \mathrm{g} / \mathrm{L} \mathrm{DNJ}$ (a) or $1 \%$ D-xylose (b). The expression 
level of each gene was normalized by that of the $g p d A$. The relative expression corresponds to the ratio of the mean expression levels of $s r p k F$ divided by that of $g p d A$. The relative expression levels are the means of three independent experiments, and the error bars indicate the standard deviations. M: MR12, filled bars; OE: OEsrpkF, striped bars. 
Fig. 1.
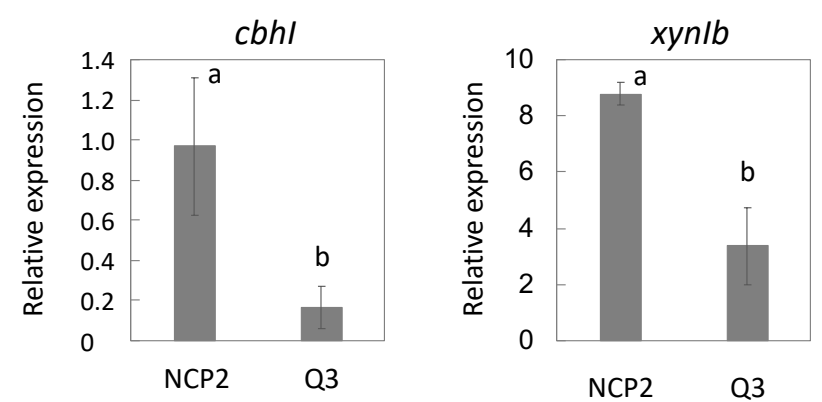

Tani et al. 
Fig. 2.

a

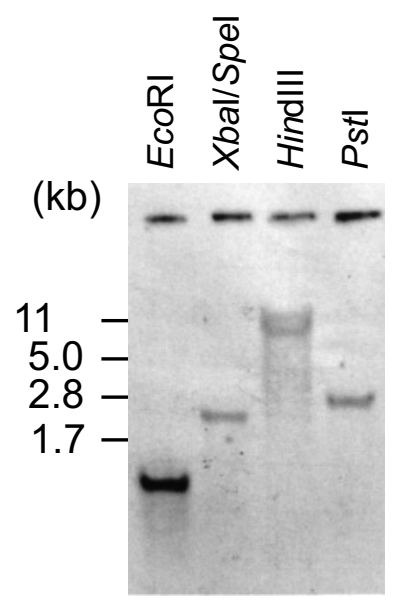

b

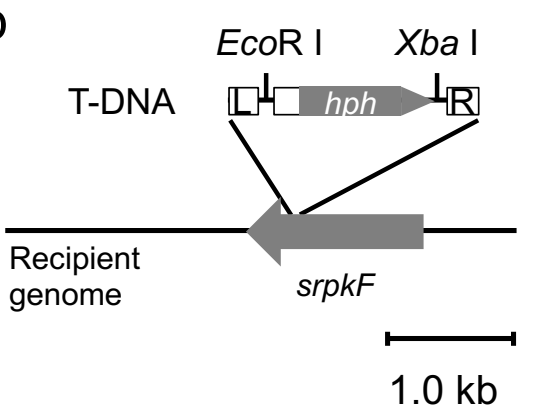

Tani et al. 
Fig. 3.

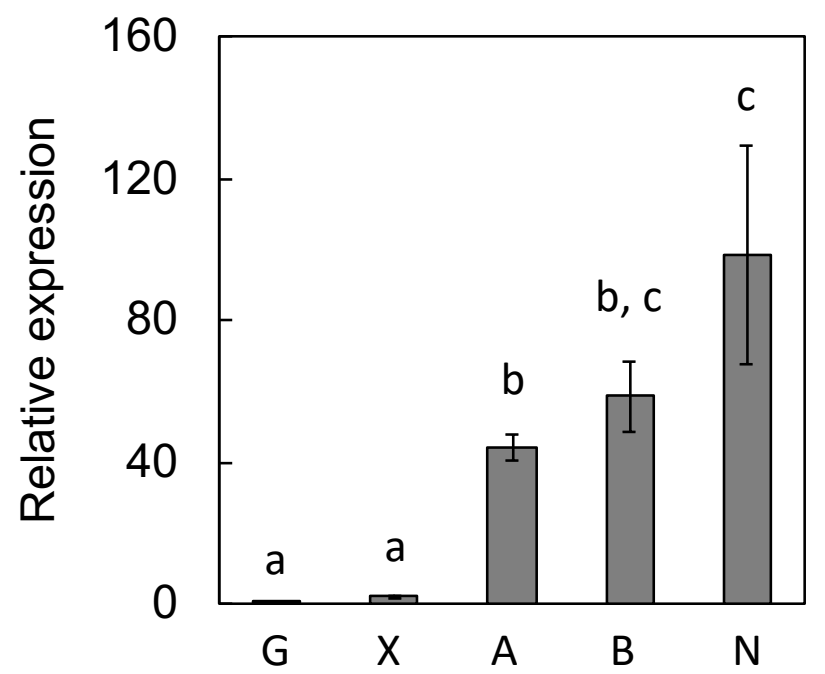

Tani et al. 
Fig. 4.
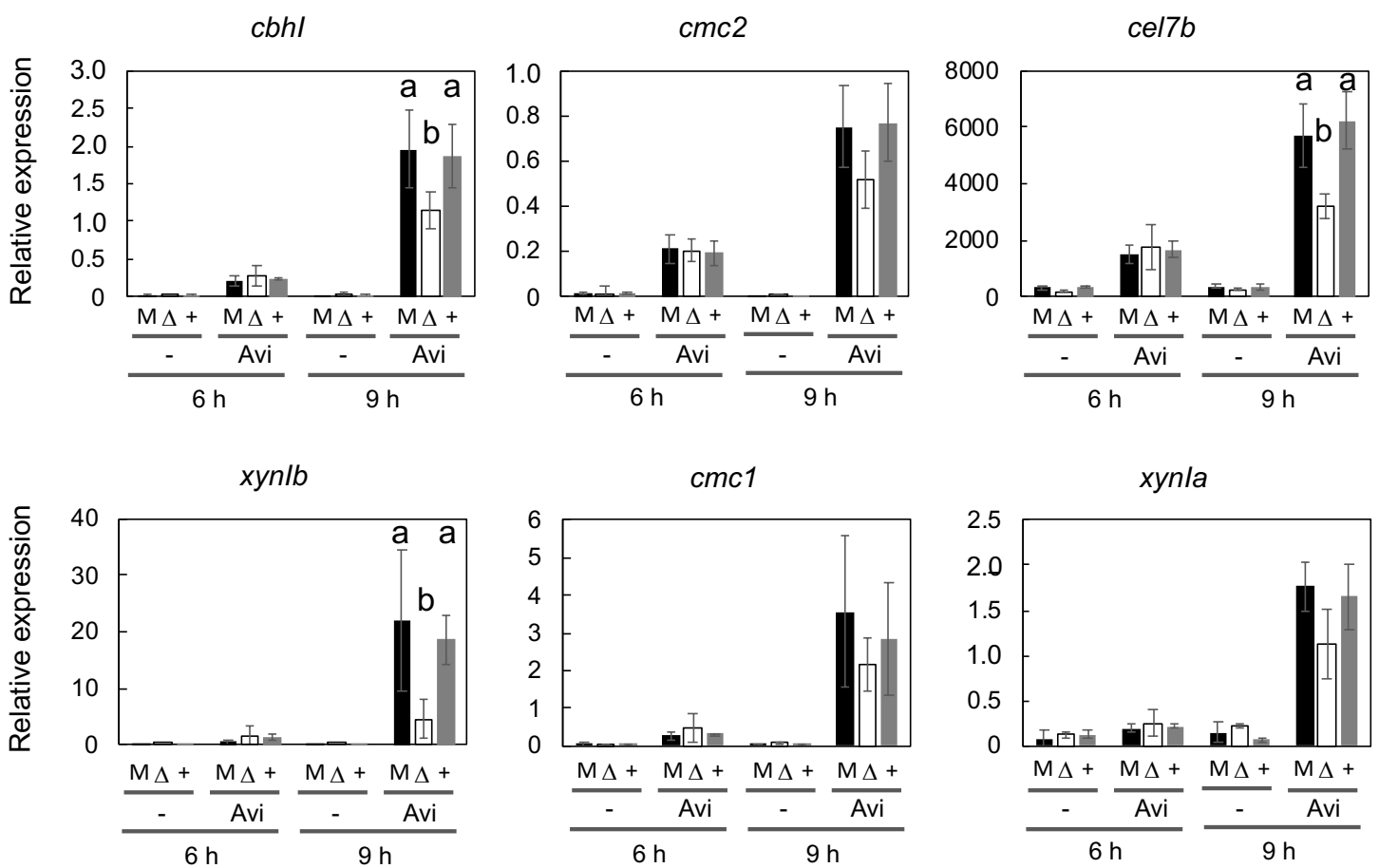

Tani et al. 
Fig. 5.
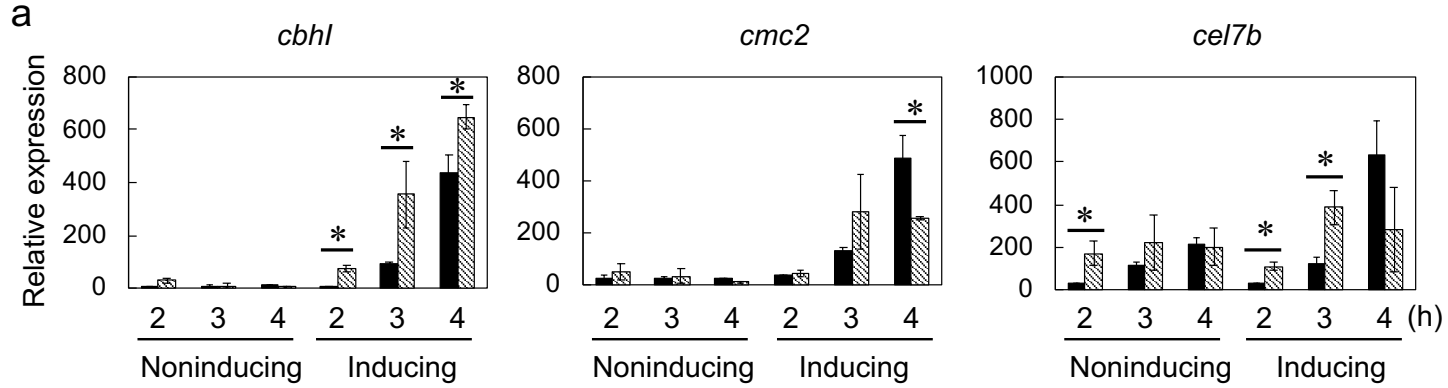

b
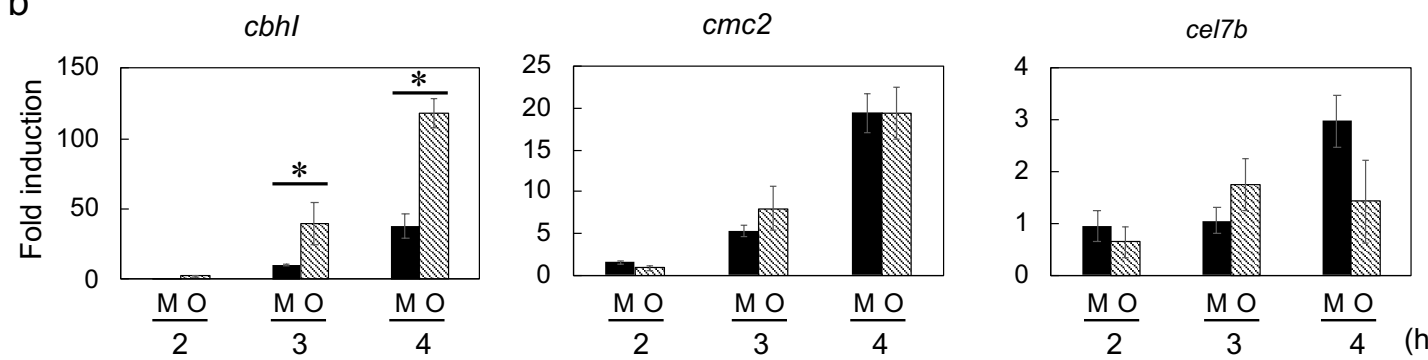

C
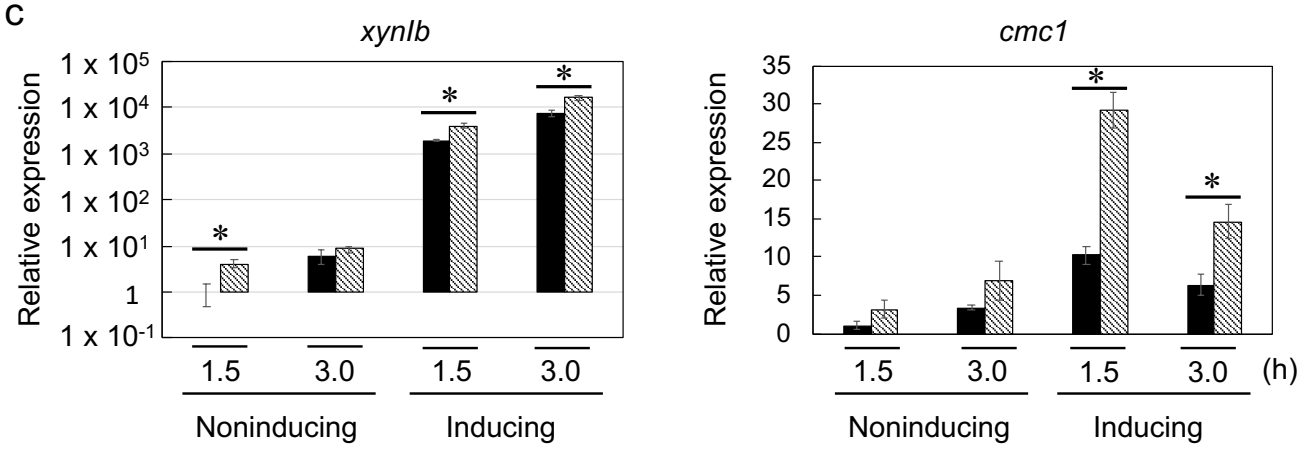

d
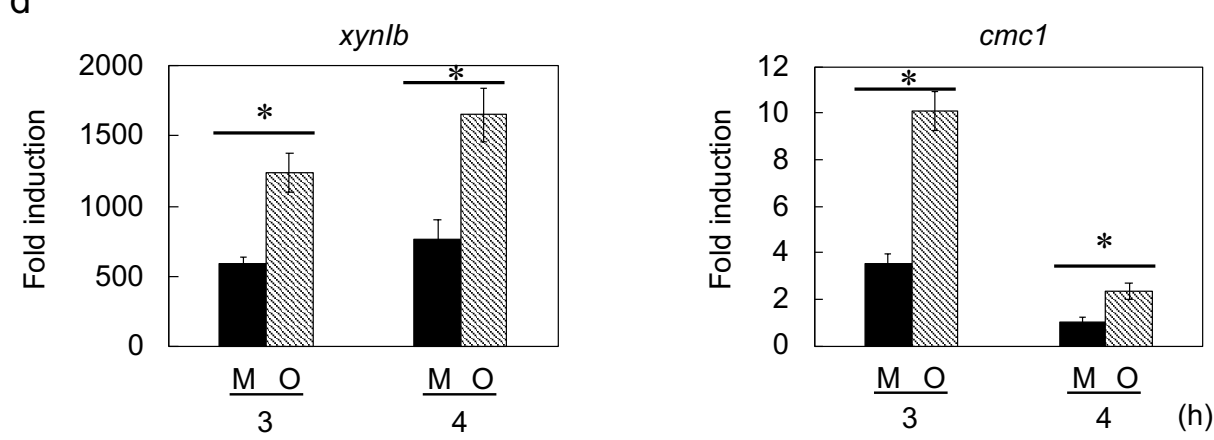

Tani et al. 
Fig. 6.

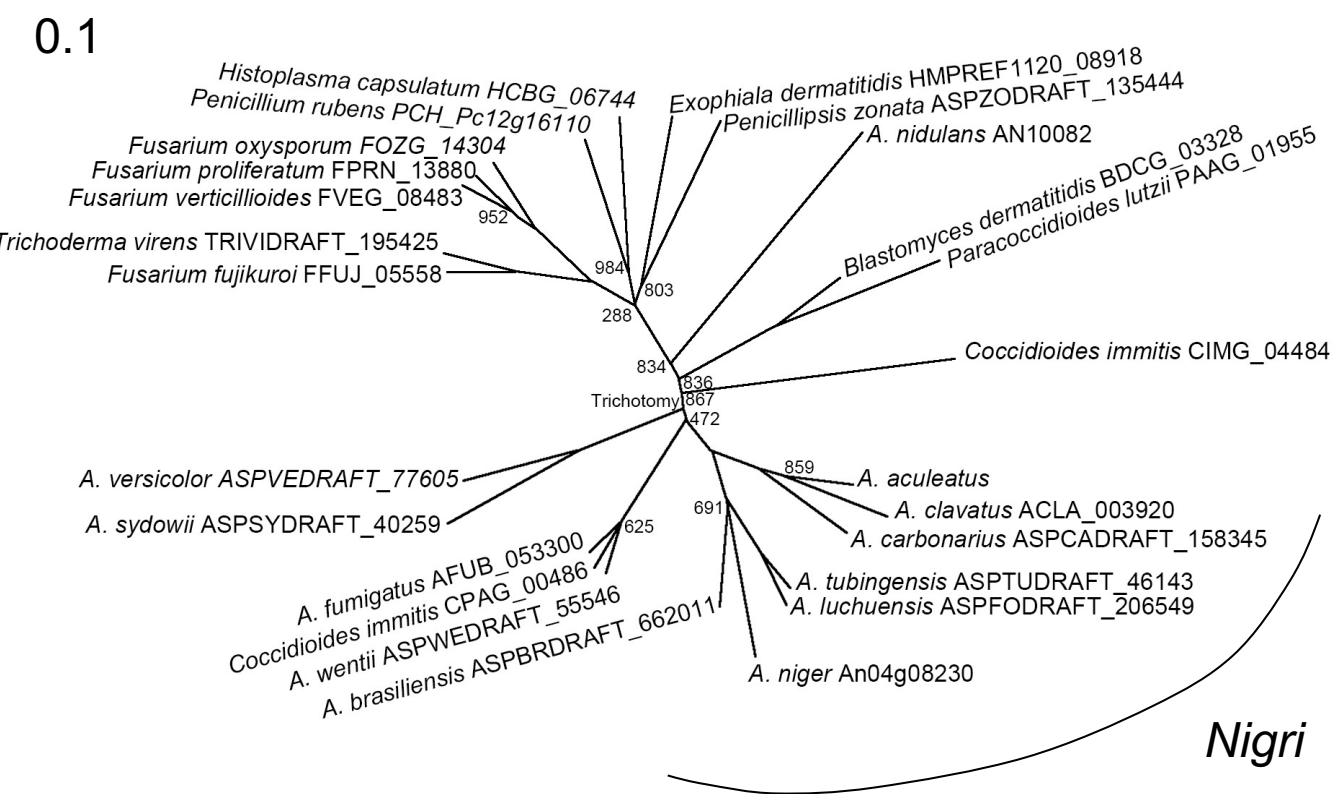

Tani et al. 
Supplementary Fig. S1.

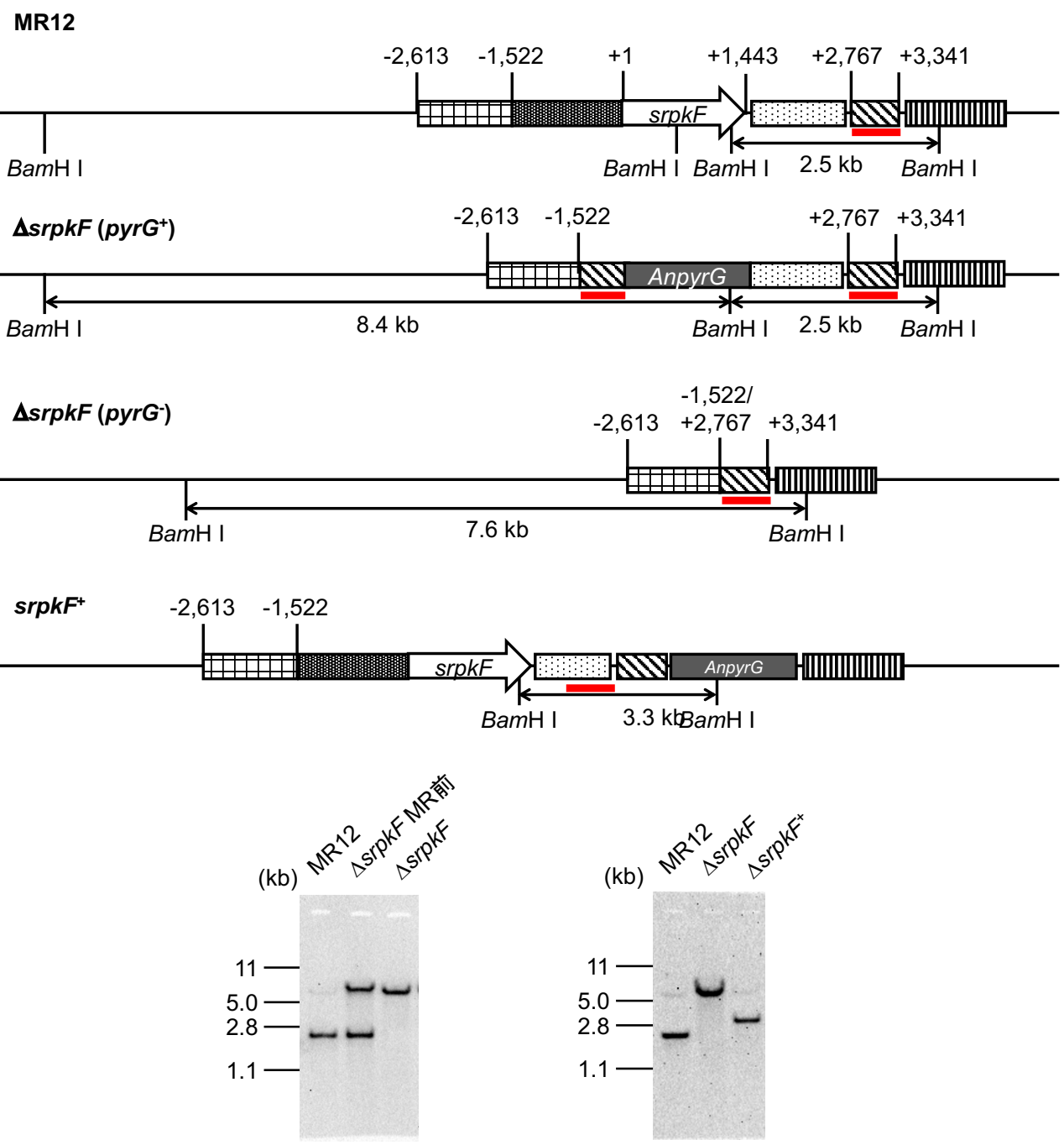

Tani et al. 
Supplementary Fig. S2.

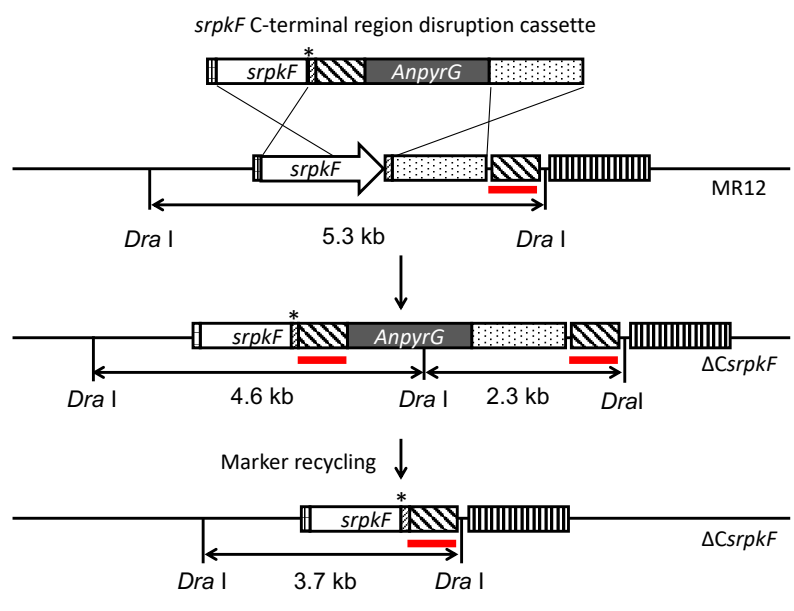

srpkF complementation cassette
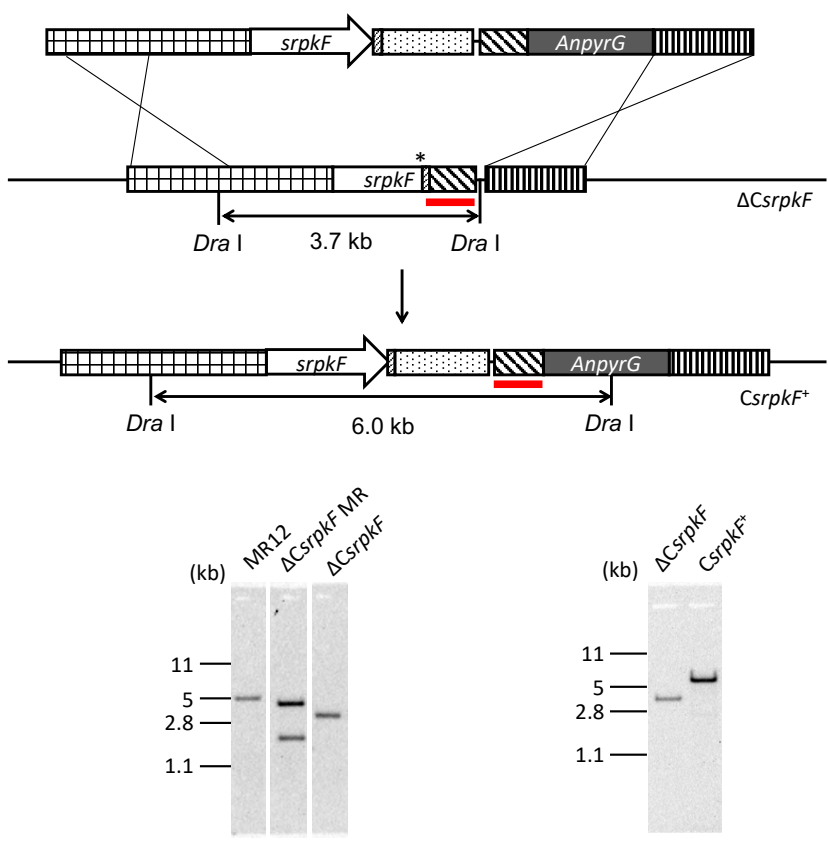

Tani et al. 
Supplementary Fig. S3.
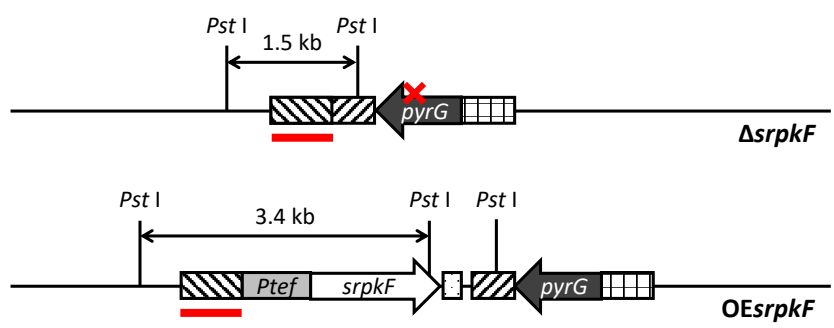

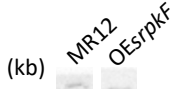

$$
\begin{aligned}
& 11- \\
& \text { 5- } \\
& 1.1-
\end{aligned}
$$

Tani et al. 
Supplementary Fig. S4.
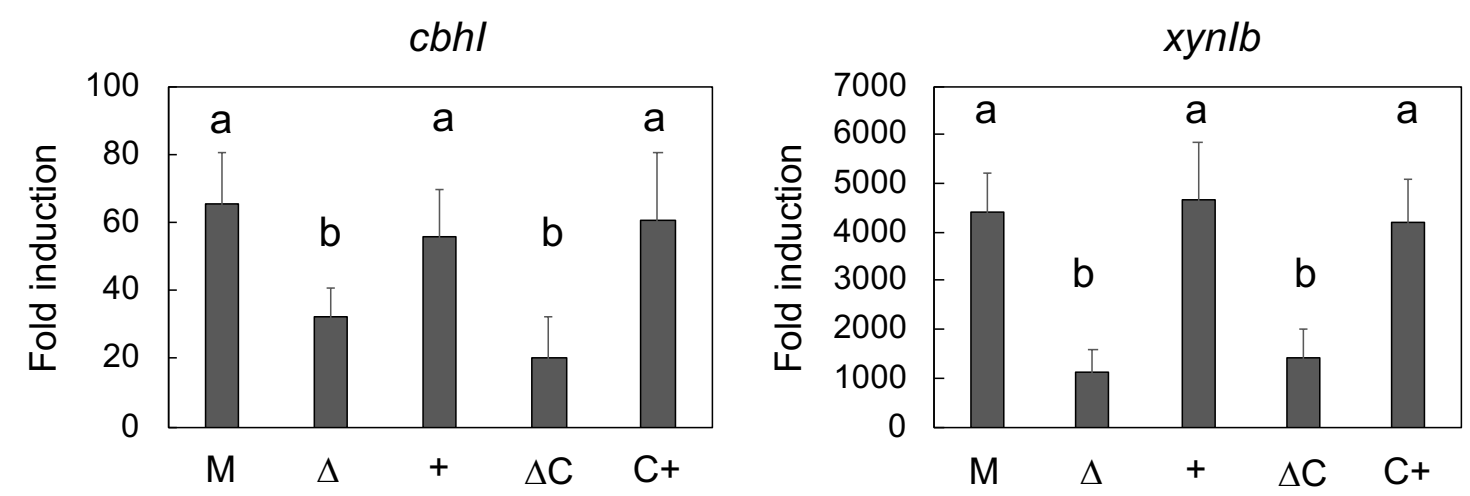

Tani et al. 
Supplementary Fig. S5.
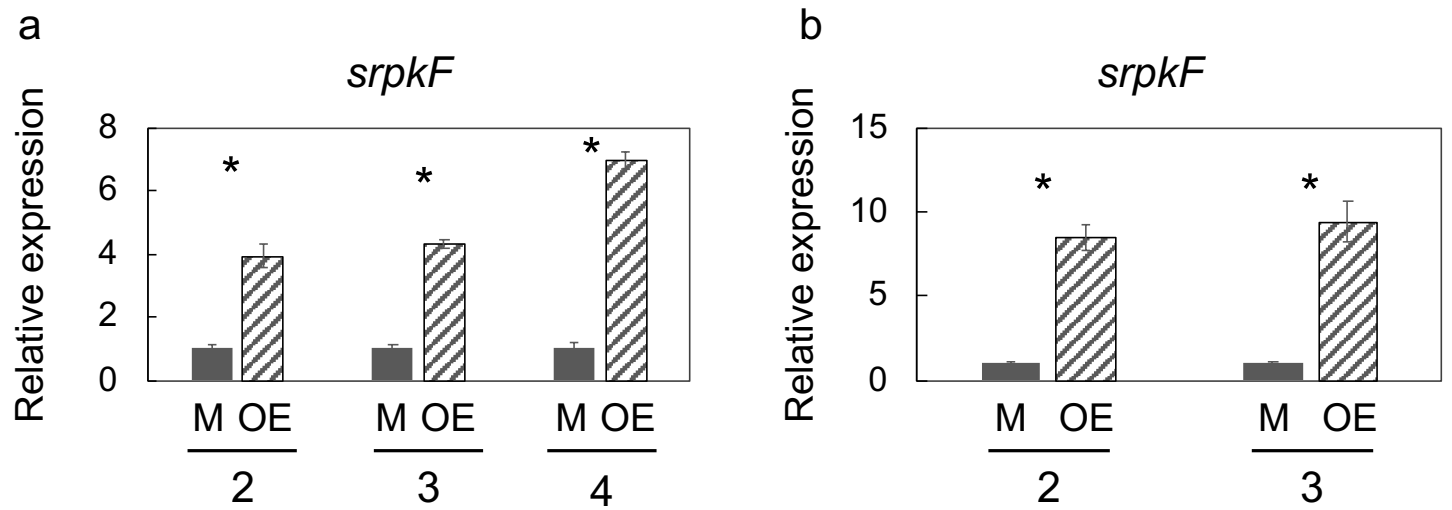

Tani et al. 